\title{
Heritability is a poor, if not unhelpful, measure of complex human behavioral processes
}

BBS commentary on Uchiyama et al., 'Cultural evolution of genetic heritability. Agustín Fuentes ${ }^{1}$, Kevin A. Bird ${ }^{2}$

1. Dept. of Anthropology, Princeton University, Aaron Burr Hall, Princeton New Jersey 08540

2. Michigan State University, Department of Horticulture and Evology, Evolution and Behavior program, 1066 Bogue St, East Lansing, MI 48824

Email: 1. afuentes2@princeton.edu (primary contact), 2. birdkevi@msu.edu

\begin{abstract}
Heritability is not a measure of the relative contribution of nature vis-à-vis nurture, nor is it the phenotypic variance explained by or due to genetic variance. Heritability is a correlative value. The evolutionary and developmental processes associated with human culture challenge the use of 'heritability' for understanding human behavior.
\end{abstract}

\section{Commentary:}

Much of what are referred to as "traits" in behavioral genetics and cultural evolution are not bounded entities or structurally and functionally individual units of transmission or specific targets of selection. Human culture, as an evolutionary and developmental process, calls into question the very meaning and significance of 'heritability' for understanding why and how humans do what they do (Fuentes 2016, Jannson et al. 2021, Lewens 2015, Rosenberg et al. 2019, Stotz 2010, Uller and Laland 2019).

Uchiyama et al.'s goal of offering a more nuanced understanding of the interaction between 'genes' and 'culture' is laudable and partially achieved. As is hinted at throughout the article, the focus on explaining the relative genetic or cultural contribution to variation in traits may be misrepresenting the actual dynamics, developmental, social/experiential, and physiological processes interacting to produce the measured target. This is particularly salient in the case of human behavioral-cognitive-developmental-culturally mediated/structured parameters, such as IQ. Figure 2 of the target article ("Predicting Heritability") identifies three key contributing processes: variability in the trait, variability in environment and culture, and variability in genes. Uchiyama et al. acknowledge that "Psychological and behavioral phenotypes are typically the outcome of a complex network of interactions that involve all these factors," but still graphically represent each of the three as if they are processually independent as analytical units. This is despite much in the article arguing against such simplistic representation. This kind of 'particulate and separate'-with a nod to "we know in reality it is not this simple"-- approach is exactly the problem at hand, and leads to substantive errors of interpretation and understanding.

The continued misuse of "heritability" facilitates such errors. Uchiyama et al. note heritability is not a "measure of the relative contribution of nature vis-à-vis nurture," but their description of it as phenotypic variance explained by or due to genetic variance is also misleading because it implies causality. Falconer (1967) notes heritability is the ratio of additive genetic variance and 
phenotypic variance and that it is the regression of breeding value on phenotypic value (Falconer, 1967 p.165). Kempthorne (1978) argues that an analysis of variance (e.g. heritability estimates from twin studies) can tell us nothing about causation and we should abandon phrasing like $\mathrm{X} \%$ of variance is "due to" $\mathrm{Y}$ and use the more accurate $\mathrm{X} \%$ of variance is "associated linearly” with Y (Kempthorne 1978). Both descriptions make clear that heritability is a correlative value. The extent to which a linear association can be interpreted as a causal relationship depends on properly controlling for confounding variables. In plant breeding this is a reasonable inference because of the ability to randomize environments with respect to genotype through experimental design and replication of genotypes (Kempthorne, 1978). In human genetics, this is not possible and several processes complicate causal interpretation of heritability estimates (Briley et al. 2019).

Genome-wide association studies and polygenic scores for human behavioral traits do not rectify the heritability problem. GWAS loci are fundamentally correlative and although the environment has no direct influence on nucleotide sequence, the processes that lead to a correlation between a genetic variant and a trait are not necessarily due to genetic causation. It is difficult to interpret polygenic scores as unconditionally providing strong information about genetic causation when estimates frequently contain effects of non-transmitted parental alleles (Kong et al. 2018), and effect sizes vary across age, sex, and socioeconomic status (Mostafavi et al. 2020). GWAS samples show inherent bias based on selective participation (Tyrell et al. 2021), and disentangling the genetic, environmental and cultural contribution to differing polygenic scores between populations is difficult or impossible (Rosenberg et al. 2019). Mathieson (2021) extends the omnigenic model, arguing that polygenic scores show differential prediction because the effects of peripheral genes (those that affect traits indirectly through interactions with other genes) differ across populations. Mathieson further argues that peripheral environmental factors (those that indirectly affect traits though interaction with other environmental factors) differ in their effect across populations. Culture provides an explanation for how this may occur since cultural factors structure and constrain the relationships between traits and environments. Uchiyama et al. use language to the effect that culture "masks" or "unmasks" genes. This implies there is a "true" genetic architecture to the trait. However, Mathieson's argument that the roles of peripheral genes and environments vary across contexts would mean that the biological and environmental underpinnings of fundamentally social traits like education or IQ change depending on culture, space, and time. In other words, the nature of the traits themselves is radically dynamic. We see this also in plant breeding where, for example, yield of corn plants is related to multiple factors like nitrogen uptake, resistance to drought, leaf angle, plant architecture, etc, but the contribution of these other traits to yield varies greatly by location and year (Tucker et al. 2020).

Culture systematically structures the types of shared and unshared environments experienced by individuals, the interactions between those shared and unshared environments, the interactions and correlations between genes and environments that can exist, and even genetic associations with a trait. Culture is a permeating confound that produces contingent, often non-causal, linear relationships between genetic relatedness and phenotypic similarity. Culture will determine the kinds of factors within a target population that influence the correlation between a genetic variant 
and a phenotype. When linear associations between genetic similarity and phenotypic similarity are this deeply confounded, the very meaning and significance of heritability estimates are called into question.

If, as Uchiyama et al. state, "Heritability is not a property of a trait in itself, because in the absence of a reference culture it is necessarily unstable" and "Nothing in behavioral genetics makes sense except in the light of cultural evolution," it stands to reason that heritability is a poor, if not unhelpful, measure for effective evaluation of the dynamics of complex human behavioral processes. One could read the core of the argument of this manuscript as tentatively making this case, and we suggest that it can, and should, be made more conclusively. 


\section{References:}

Briley, D. A., Livengood, J., Derringer, J., Tucker-Drob, E. M., Fraley, R. C., \& Roberts, B. W. (2019). Interpreting behavior genetic models: seven developmental processes to understand. Behavior genetics, 49(2), 196-210.

Falconer, D. S. (1967). Introduction to quantitative genetics. Oliver and Boys Ltd. London. P. Jansson F, Aguilar E, Acerbi A, Enquist M. 2021 Modelling cultural systems and selective filters. Phil. Trans. R. Soc. B 376: 20200045. https://doi.org/10.1098/rstb.2020.0045

Kempthorne, O. (1978). A biometrics invited paper: Logical, epistemological and statistical aspects of nature-nurture data interpretation. Biometrics, 1-23.

Kong, A., Thorleifsson, G., Frigge, M. L., Vilhjalmsson, B. J., Young, A. I., Thorgeirsson, T. E., ... \& Stefansson, K. (2018). The nature of nurture: Effects of parental genotypes. Science, 359(6374), 424-428.

Lewens T. (2015). Cultural Evolution: Conceptual Challenges. Oxford, UK: Oxford University Press.

Mathieson, I. (2021). The omnigenic model and polygenic prediction of complex traits. The American Journal of Human Genetics. https://doi.org/10.1016/j.ajhg.2021.07.003.

Mostafavi, H., Harpak, A., Agarwal, I., Conley, D., Pritchard, J. K., \& Przeworski, M. (2020). Variable prediction accuracy of polygenic scores within an ancestry group. Elife, 9, e48376.

Rosenberg, N. A., Edge, M. D., Pritchard, J. K., \& Feldman, M. W. (2019). Interpreting polygenic scores, polygenic adaptation, and human phenotypic differences. Evolution, medicine, and public health, 2019(1), 26-34.

Stotz, K. (2010). Human nature and cognitive-developmental niche construction.

Phenomenology and Cognitive Science 9 483-501.

Tucker, S. L., Dohleman, F. G., Grapov, D., Flagel, L., Yang, S., Wegener, K. M., ... \& Rice, E. A. (2020). Evaluating maize phenotypic variance, heritability, and yield relationships at multiple biological scales across agronomically relevant environments. Plant, cell \& environment, 43(4), 880-902.

Tyrrell, J., Zheng, J., Beaumont, R., Hinton, K., Richardson, T. G., Wood, A. R., ... \& Tilling, K. (2021). Genetic predictors of participation in optional components of UK Biobank. Nature communications, 12(1), 1-13.

Uller, T. and Laland, K. N. (2019) Evolutionary Causation: Biological and philosophical reflections. Cambridge, MA; MIT Press. 
